\title{
ON PARAMETRIC EXTENSIONS OVER NUMBER FIELDS
}

\author{
FRANÇOIS LEGRAND
}

\begin{abstract}
Given a number field $F$, a finite group $G$ and an indeterminate $T, a$ G-parametric extension over $F$ is a finite Galois extension $E / F(T)$ with Galois group $G$ and $E / F$ regular that has all the Galois extensions of $F$ with Galois group $G$ among its specializations. We are mainly interested in producing non- $G$-parametric extensions, which relates to classical questions in inverse Galois theory like the Beckmann-Black problem. Building on a strategy developed in previous papers, we show that there exists at least one non- $G$-parametric extension over $F$ for a given non-trivial finite group $G$ and a given number field $F$ under the sole necessary condition that $G$ occurs as the Galois group of a Galois extension $E / F(T)$ with $E / F$ regular.
\end{abstract}

\section{INTRODUCTION}

Given a number field $F$, the inverse Galois problem over $F$ asks whether every finite group $G$ occurs as the Galois group of a Galois extension of $F$. A classical way to obtain such an extension consists in introducing an indeterminate $T$ and in producing a Galois extension $E / F(T)$ with the same Galois group and $E / F$ regular $^{1}$ : from the Hilbert irreducibility theorem, the extension $E / F(T)$ has infinitely many linearly disjoint specializations with Galois group $G$ (if $G$ is not trivial). We refer to $\S 2.1$ for basic terminology.

Following recent works [Leg16, §4] [Leg15], we are interested in the present paper in finite Galois extensions $E / F(T)$ with $E / F$ regular from now on, say for short that the extension $E / F(T)$ is an " $F$-regular Galois extension" - that have all the Galois extensions of $F$ with Galois group $G$ among their specializations. More precisely, let us recall the following definition.

Definition 1.1. A finite $F$-regular Galois extension $E / F(T)$ with Galois group $G$ is $G$-parametric over $F$ if every Galois extension of $F$ with Galois group $G$ occurs as a specialization of $E / F(T)$.

Date: June 11, 2018.

$1_{\text {i.e. }, ~} E \cap \overline{\mathbb{Q}}=F$. 
Parametric extensions have been introduced with the aim of a better understanding of the Beckmann-Black problem which asks whether the specialization process to solve the inverse Galois problem is optimal. Namely, recall that the Beckmann-Black problem, for the finite group $G$ over the number field $F$, asks whether every Galois extension $L / F$ with Galois group $G$ is a specialization of some $F$-regular Galois extension $E_{L} / F(T)$ (possibly depending on $L / F$ ) with Galois group $G$. Although no counter-example is known and only a few positive results have been proved (see e.g. [Dèb01, Theorem 2.2] for more details), it may be expected that the Beckmann-Black problem fails in general over number fields. However, no line of attack seems to be known and a disproof is probably out of reach at the moment.

Actually, the answer to the following weaker question on parametric extensions seems to be unavailable in the literature. Say that a finite group $G$ is a "regular Galois group over (the given number field) $F$ " if $G$ occurs as the Galois group of an $F$-regular Galois extension of $F(T)$.

Question 1.2. Does there exist a regular Galois group $G$ over $F$ such that no F-regular Galois extension of $F(T)$ with Galois group $G$ is $G$-parametric over F?

The existence of such a finite group $G$ would be a first step towards a counter-example to the Beckmann-Black problem over the number field $F$. However, although we may expect the answer to be negative almost always, deciding whether a given $F$-regular Galois extension of $F(T)$ with Galois group $G$ is $G$-parametric over $F$ or not is a difficult problem in general (even in the easiest case $G=\mathbb{Z} / 2 \mathbb{Z}$ ) and only a few non-parametric extensions are available in the literature. In particular, finding a group $G$ as in Question 1.2 seems to be difficult as well.

In [Leg16, §4] and [Leg15], we offer a systematic approach to produce $F$-regular Galois extensions $E / F(T)$ with Galois group $G$ which are not $G$-parametric over $F$. It consists in introducing another $F$-regular Galois extension $E^{\prime} / F(T)$ with Galois group $G$ and in giving criteria ensuring that some specializations of $E^{\prime} / F(T)$ with Galois group $G$ are not specializations of $E / F(T)$. Examples with specific finite groups $G$ such as abelian groups, symmetric and alternating groups, non-abelian simple groups, etc. are then given, under some natural necessary conditions. For example, an obvious obstruction to the existence of at least one non- $G$-parametric extension over $F$ is that $G$ is not a regular Galois group over $F$.

Building on this strategy, we show in this paper that the latter obstruction is the only one to the existence of a non- $G$-parametric extension over $F$ with Galois group $G$. 
Theorem 1.3. Let $G$ be a non-trivial finite group and $F$ a number field. Assume that $G$ is a regular Galois group over $F$. Then there exists at least one non-G-parametric extension over $F$ with Galois group $G$.

Actually, from any $F$-regular Galois extension $E / F(T)$ with Galois group $G$ satisfying some mild assumptions on its set of branch points, we derive a sequence $\left(E_{k} / F(T)\right)_{k}$ of $F$-regular realizations of $G$ such that infinitely many linearly disjoint specializations of $E / F(T)$ with Galois group $G$ are not specializations of $E_{k} / F(T)$. See Theorem 3.1.

The paper is organized as follows. In $\S 2$, we recall some material used in the sequel. In $\S 3$, we prove Theorem 1.3 under an auxiliary result on prime divisors of polynomials (Definition 2.1) that has its own interest; see Proposition 3.5. Proposition 3.5 is proved in $\S 4$. Finally, in $\S 5$, we make related previous results from [Leg15] more precise thanks to a group theoretic argument communicated to us by Reiter.

Acknowledgments. This work was motivated by a visit of the author in Universität Bayreuth. The author is then indebted with Stefan Reiter for Lemma 5.3 and would like to thank the Zahlentheorie team for hospitality and financial support. The author also wishes to thank Lior Bary-Soroker, Pierre Dèbes, Danny Neftin and Jack Sonn for helpful discussions, as well as the anonymous referee for suggesting a simpler proof of Proposition 3.5. This research is partially supported by the Israel Science Foundation (grants No. 40/14 and No. 696/13).

\section{BASICS}

For this section, let $F$ be a number field.

2.1. Specializations of finite Galois extensions of $F(T)$. Given an indeterminate $T$, let $E / F(T)$ be a finite Galois extension with Galois group $G$ and $E / F$ regular (i.e., $E \cap \overline{\mathbb{Q}}=F$ ). From now on, say for short that $E / F(T)$ is an " $F$-regular Galois extension".

Recall that a point $t_{0} \in \mathbb{P}^{1}(\overline{\mathbb{Q}})$ is a branch point of $E / F(T)$ if the prime ideal $\left(T-t_{0}\right) \overline{\mathbb{Q}}\left[T-t_{0}\right]^{2}$ ramifies in the integral closure of $\overline{\mathbb{Q}}\left[T-t_{0}\right]$ in the compositum of $E$ and $\overline{\mathbb{Q}}(T)$ (in a fixed algebraic closure of $F(T)$ ). The extension $E / F(T)$ has only finitely many branch points.

Given a point $t_{0} \in \mathbb{P}^{1}(F)$, not a branch point, the residue extension of $E / F(T)$ at a prime ideal $\mathcal{P}$ lying over $\left(T-t_{0}\right) F\left[T-t_{0}\right]$ is denoted by $E_{t_{0}} / F$ and called the specialization of $E / F(T)$ at $t_{0}$. It does not depend on the choice of the prime $\mathcal{P}$ lying over $\left(T-t_{0}\right) F\left[T-t_{0}\right]$ as $E / F(T)$ is Galois. The extension $E_{t_{0}} / F$ is Galois with Galois group a subgroup of $G$, namely the decomposition group of $E / F(T)$ at $\mathcal{P}$.

\footnotetext{
${ }^{2}$ Replace $T-t_{0}$ by $1 / T$ if $t_{0}=\infty$.
} 
2.2. Prime divisors of polynomials. Denote the integral closure of $\mathbb{Z}$ in $F$ by $O_{F}$. Let $P(T) \in O_{F}[T]$ be a non-constant monic polynomial.

Definition 2.1. Say that a non-zero prime ideal $\mathcal{P}$ of $O_{F}$ is a prime divisor of $P(T)$ if the reduction of $P(T)$ modulo $\mathcal{P}$ has a root in the residue field $O_{F} / \mathcal{P}$.

The following lemma will be used on several occasions in the sequel. Denote the roots of $P(T)$ by $t_{1}, \ldots, t_{r}$. Given an integer $k \geq 1$ and an index $j \in\{1, \ldots, r\}$, let $\sqrt[k]{t_{j}}$ be a $k$-th root of $t_{j}$. Finally, let $L_{k}$ be the splitting field of $P\left(T^{k}\right)$ over $F$ and $\zeta_{k}$ a primitive $k$-th root of unity.

Lemma 2.2. The following three conditions are equivalent:

(1) $\bigcup_{j=1}^{r} \bigcup_{l=0}^{k-1} \operatorname{Gal}\left(L_{k} / F\left(\zeta_{k}^{l} \sqrt[k]{t_{j}}\right)\right) \neq \bigcup_{j=1}^{r} \operatorname{Gal}\left(L_{k} / F\left(t_{j}\right)\right)$,

(2) there exists a set $\mathcal{S}$ of non-zero prime ideals of $O_{F}$ that has positive density and such that each prime ideal $\mathcal{P}$ in $\mathcal{S}$ is a prime divisor of $P(T)$ but not of $P\left(T^{k}\right)$,

(3) there exist infinitely many non-zero prime ideals of $O_{F}$ each of which is a prime divisor of $P(T)$ but not of $P\left(T^{k}\right)$.

Proof. We may assume that $P(T)$ is separable. If $P(0)=0$, then (1), $(2)$ and (3) fail. From now on, we assume that $P(0) \neq 0$. In particular, $P\left(T^{k}\right)$ is separable.

First, assume that (1) holds, i.e., there exists some $\sigma$ in

$$
\bigcup_{j=1}^{r} \operatorname{Gal}\left(L_{k} / F\left(t_{j}\right)\right) \backslash \bigcup_{j=1}^{r} \bigcup_{l=0}^{k-1} \operatorname{Gal}\left(L_{k} / F\left(\zeta_{k}^{l} \sqrt[k]{t_{j}}\right)\right) .
$$

By the Tchebotarev density theorem, there exists a positive density set $\mathcal{S}$ of primes $\mathcal{P}$ of $O_{F}$ such that the associated Frobenius in $L_{k} / F$ is conjugate to $\sigma$. As $\sigma$ fixes no root of $P\left(T^{k}\right)$, such a $\mathcal{P}$ is not a prime divisor of $P\left(T^{k}\right)$ (up to finitely many). Denote the splitting field of $P(T)$ over $F$ by $L_{1}$. Then the Frobenius associated with $\mathcal{P}$ in $L_{1} / F$ is the restriction to $L_{1}$ of the one in $L_{k} / F$. As $\sigma$ fixes a root of $P(T), \mathcal{P}$ is a prime divisor of $P(T)$ (up to finitely many), as needed for (2).

As implication $(2) \Rightarrow(3)$ is obvious, it remains to prove implication $(3) \Rightarrow(1)$. To do this, assume that (1) does not hold. Let $\mathcal{P}$ be a non-zero prime ideal of $O_{F}$ that is a prime divisor of $P(T)$ and that is unramified in $L_{k} / F$. Denote the associated Frobenius in $L_{k} / F$ by $\sigma$. As $\mathcal{P}$ is a prime divisor of $P(T)$ and $\mathcal{P}$ does not ramify in $L_{1} / F$, the associated Frobenius in $L_{1} / F$ fixes a root of $P(T)$ (up to finitely many). Since this Frobenius is the restriction of $\sigma$ to $L_{1}$, we get that $\sigma$ fixes a root of $P(T)$. As condition (1) fails, $\sigma$ fixes a root of $P\left(T^{k}\right)$ as 
well. Hence $\mathcal{P}$ is a prime divisor of $P\left(T^{k}\right)$ (up to finitely many). Then (3) does not hold either, thus ending the proof.

\section{Proof of Theorem 1.3}

The aim of this section consists in proving Theorem 3.1 below whose Theorem 1.3 is a straightforward application.

3.1. Statement of Theorem 3.1. Let $F$ be a number field, $O_{F}$ the integral closure of $\mathbb{Z}$ in $F$ and $G$ a non-trivial finite group that is a regular Galois group over $F$ (i.e., $G$ occurs as the Galois group of an $F$-regular Galois extension of $F(T))$.

Given an indeterminate $T$, let $E / F(T)$ be an $F$-regular Galois extension with Galois group $G$, branch points $t_{1}, \ldots, t_{r}$ and such that the following two conditions hold ${ }^{3}$ :

(bp-1) $\{0,1, \infty\} \cap\left\{t_{1}, \ldots, t_{r}\right\}=\emptyset$,

(bp-2) $t_{1}, \ldots, t_{r}$ all are integral over $O_{F}$.

Theorem 3.1. There exists a sequence of $F$-regular Galois extensions $E_{k} / F(T), k \in \mathbb{N} \backslash\{0\}$ (depending on $E / F(T)$ ), with Galois group $G$ and that satisfies the following conclusion.

For each finite extension $F^{\prime} / F$, there exist infinitely many positive integers $k$ (depending on $F^{\prime}$ ) such that the extension $E_{k} F^{\prime} / F^{\prime}(T)$ satisfies the following condition:

(non-G-parametricity) there exist infinitely many linearly disjoint Galois extensions of $F^{\prime}$ with Galois group $G$ each of which is not a specialization of $E_{k} F^{\prime} / F^{\prime}(T)$.

In particular, the extension $E_{k} F^{\prime} / F^{\prime}(T)$ is not $G$-parametric over $F^{\prime}$. Furthermore, these Galois extensions of $F^{\prime}$ with Galois group $G$ may be produced by specializing the extension $E F^{\prime} / F^{\prime}(T)$.

Remark 3.2. (1) As a classical consequence of the Riemann existence theorem, every finite group $G$ is a regular Galois group over some number field $F_{G}$, and then over every number field $F^{\prime}$ containing $F_{G}$. Hence Theorem 3.1 provides the following statement.

Let $G$ be a non-trivial finite group. Then there exists some number field $F_{G}$ that satisfies the following property. For each number field $F^{\prime}$ containing $F_{G}$, there exists an $F^{\prime}$-regular Galois extension of $F^{\prime}(T)$ with Galois group $G$ which satisfies the (non- $G$-parametricity) condition. Moreover, one can take $F_{G}$ equal to a given number field $F$ if and only if $G$ is a regular Galois group over $F$.

\footnotetext{
${ }^{3}$ These two conditions hold up to applying a suitable change of variable.
} 
(2) As explained in $§ 3.2 .4$ below, we are not able to remove the dependence on the number field $F^{\prime}$ containing $F$ in the set of all suitable positive integers $k$. In particular, the proof provides no integer $k$ such that the extension $E_{k} F^{\prime} / F^{\prime}(T)$ satisfies the (non- $G$-parametricity) condition for each finite extension $F^{\prime} / F$. See Proposition 5.1 for a result with such a geometric conclusion.

3.2. Proof of Theorem 3.1. We break the proof into four parts.

3.2.1. Notation. Given a positive integer $k$ and $j \in\{1, \ldots, r\}$, let $\sqrt[k]{t_{j}}$ be a $k$-th root of $t_{j}$. Let $F^{\prime} / F$ be a finite extension and $O_{F^{\prime}}$ the integral closure of $\mathbb{Z}$ in $F^{\prime}$.

By condition (bp-1), one may consider the polynomial

$$
P_{E}(T):=\prod_{j=1}^{r}\left(T-t_{j}\right) .
$$

By condition (bp-2), the monic separable polynomial $P_{E}(T)$ has coefficients in $O_{F}$.

\subsubsection{Two lemmas. Fix a positive integer $k$.}

First, we derive from the extension $E / F(T)$ an $F$-regular Galois extension of $F(T)$ with group $G$ and specified set of branch points.

Lemma 3.3. There exists an F-regular Galois extension of $F(T)$ with Galois group $G$ and whose branch points are exactly the $k$-th roots of those of $E / F(T)$.

Proof. The proof below follows part of an argument of Dèbes and Zannier given in the proof of [DW08, Proposition 5.2]. Let $P(T, Y) \in$ $F[T][Y]$ be the irreducible polynomial over $F(T)$ of some primitive element of $E$ over $F(T)$, assumed to be integral over $F[T]$. The polynomial $P(T, Y)$ is absolutely irreducible (as $E / F(T)$ is $F$-regular) and, as 0 is not a branch point (condition (bp-1)), it has a root in $\overline{\mathbb{Q}}((T))$. By [Dèb92, Lemma 0.1], the polynomial $P_{k}(T, Y):=P\left(T^{k}, Y\right)$ is absolutely irreducible. Denote the field generated by one root of $P_{k}(T, Y)$ over $F(T)$ by $E_{k}$. The extension $E_{k} / F(T)$ is $F$-regular (as $P_{k}(T, Y)$ is absolutely irreducible) and has degree equal to the order of $G$. Denote the Galois closure of $E_{k} / F(T)$ by $\widehat{E_{k}} / F(T)$ and the Galois group of $\widehat{E_{k}} / F(T)$ by $H_{k}$. By the Hilbert irreducibility theorem, there are infinitely many $t_{0} \in F$ such that the specialization $\left(\widehat{E_{k}}\right)_{t_{0}} / F$ of $\widehat{E_{k}} / F(T)$ at $t_{0}$ has Galois group $H_{k}$. For all but finitely many $t_{0} \in F$, the field $\left(\widehat{E_{k}}\right)_{t_{0}}$ is the splitting field over $F$ of the polynomial $P_{k}\left(t_{0}, Y\right)=P\left(t_{0}^{k}, Y\right)$, which is in turn the field $E_{t_{0}^{k}}$. Hence there is a specialization of $E / F(T)$ 
with Galois group $H_{k}$. In particular, $H_{k}$ is a subgroup of $G$. As the order of $G$ divides the order of $H_{k}$, we get $G=H_{k}$. Hence $E_{k} / F(T)$ is an $F$-regular Galois extension with Galois group $G$. By construction, the branch points of $E_{k} / F(T)$ lying in $\overline{\mathbb{Q}} \backslash\{0\}$ are the $k$-th roots of those of $E / F(T)$. As neither 0 nor $\infty$ is a branch point of $E / F(T)$ (condition (bp-1)), the same is true of $E_{k} / F(T)$, thus ending the proof.

Let $E_{k} / F(T)$ be an $F$-regular Galois extension with Galois group $G$ and whose branch points are exactly the $k$-th roots of those of $E / F(T)$.

Next, we apply a previous criterion from [Leg16] for the extension $E_{k} F^{\prime} / F^{\prime}(T)$ to satisfy the (non- $G$-parametricity) condition.

Lemma 3.4. Assume that there exist infinitely many non-zero prime ideals of $O_{F^{\prime}}$ each of which is a prime divisor of $P_{E}(T)$ but not of $P_{E}\left(T^{k}\right)$ (considered as polynomials with coefficients in $\left.F^{\prime}\right)$. Then the extension $E_{k} F^{\prime} / F^{\prime}(T)$ satisfies the (non- $G$-parametricity) condition. Moreover, the Galois extensions of $F^{\prime}$ with Galois group $G$ appearing in the (non-G-parametricity) condition may be produced by specializing the extension $E F^{\prime} / F^{\prime}(T)$.

Proof. Given an algebraic number $t \neq 0$, denote the irreducible polynomial of $t$ over $F^{\prime}$ by $m_{t}(T)$. Consider the following four polynomials:

$$
\begin{gathered}
m_{E F^{\prime}}(T)=\prod_{j=1}^{r} m_{t_{j}}(T), \\
m_{E F^{\prime}}^{*}(T)=\prod_{j=1}^{r} m_{1 / t_{j}}(T), \\
m_{E_{k} F^{\prime}}(T)=\prod_{j=1}^{r} \prod_{l=0}^{k-1} m_{e^{2 i \pi l / k} \sqrt[k]{t_{j}}}(T), \\
m_{E_{k} F^{\prime}}^{*}(T)=\prod_{j=1}^{r} \prod_{l=0}^{k-1} m_{1 /\left(e^{2 i \pi l / k} \sqrt[k]{t_{j}}\right)}(T) .
\end{gathered}
$$

By [Leg16, Theorem 4.2] and since the branch points of the extension $E_{k} / F(T)$ are the $k$-th roots of those of $E / F(T)$, it suffices to prove that there exist infinitely many non-zero prime ideals of $O_{F^{\prime}}$ each of which is a prime divisor of $m_{E F^{\prime}}(T) \cdot m_{E F^{\prime}}^{*}(T)$ but not of $m_{E_{k} F^{\prime}}(T) \cdot m_{E_{k} F^{\prime}}^{*}(T)$.

As $\infty$ is not a branch point of $E F^{\prime} / F^{\prime}(T)$ (condition (bp-1)), one may apply [Leg16, Remark 3.11] to get that $m_{E F^{\prime}}(T) \cdot m_{E F^{\prime}}^{*}(T)$ and $m_{E F^{\prime}}(T)$ have the same prime divisors (up to finitely many). Since the polynomials $m_{E F^{\prime}}(T)$ and $P_{E}(T)$ have the same prime divisors, we get that $m_{E F^{\prime}}(T) \cdot m_{E F^{\prime}}^{*}(T)$ and $P_{E}(T)$ have the same prime divisors 
(up to finitely many). By the same argument, every prime divisor of $m_{E_{k} F^{\prime}}(T) \cdot m_{E_{k} F^{\prime}}^{*}(T)$ is a prime divisor of $P_{E}\left(T^{k}\right)$ (up to finitely many). Then, from the assumption in the statement, there exist infinitely many non-zero prime ideals of $O_{F^{\prime}}$ each of which is a prime divisor of $m_{E F^{\prime}}(T) \cdot m_{E F^{\prime}}^{*}(T)$ but not of $m_{E_{k} F^{\prime}}(T) \cdot m_{E_{k} F^{\prime}}^{*}(T)$, as needed.

3.2.3. A number theoretical result. Now, we need the following number theoretical result to ensure that the assumption of Lemma 3.4 holds.

Proposition 3.5. Given a monic separable polynomial $P(T) \in O_{F}[T]$ such that $P(0) \neq 0$ and $P(1) \neq 0$, there is an infinite set $S$ of integers $k \geq 1$ such that, for each $k \in S$, there are infinitely many prime ideals of $O_{F}$ each of which is a prime divisor of $P(T)$ but not of $P\left(T^{k}\right)$.

Remark 3.6. (1) If either 0 or 1 is a root of $P(T)$, then the conclusion of Proposition 3.5 clearly fails.

(2) The set $S$ depends on the polynomial $P(T)$ and this dependence cannot be removed. Indeed, given an integer $k \geq 1$, all non-zero prime ideals of $O_{F}$ are prime divisors of $P(T)$ and $P\left(T^{k}\right)$ if $P(T)=T-2^{k}$.

(3) Similarly, the set $S$ depends on the number field $F$ and this dependence cannot be removed. Indeed, given a number field $F^{\prime}$ containing $F$ and an integer $k \geq 1$, all but finitely many prime ideals of $O_{F^{\prime}}$ are prime divisors of $P(T)$ and $P\left(T^{k}\right)$ if $F^{\prime}$ contains a root of $P\left(T^{k}\right)$.

Proposition 3.5 is proved in $\S 4$.

3.2.4. Conclusion. As already said, the monic separable polynomial $P_{E}(T)$ has coefficients in $O_{F^{\prime}}$. Moreover, by condition (bp-1), one has $P_{E}(0) \neq 0$ and $P_{E}(1) \neq 0$. Then, by Proposition 3.5 (applied over $F^{\prime}$ ), there exists an infinite set $S$ of positive integers $k$ (depending on $F^{\prime}$; see Remark 3.6) such that, for each $k \in S$, there exist infinitely many non-zero prime ideals of $O_{F^{\prime}}$ each of which is a prime divisor of $P_{E}(T)$ but not of $P_{E}\left(T^{k}\right)$. It then remains to apply Lemma 3.4 to conclude.

\section{Proof of Proposition 3.5}

This section is organized as follows. In $\S 4.1$, we state Proposition 4.1 which is Proposition 3.5 for polynomials whose roots all are in the base number field. Next, we explain in $\S 4.2$ how deducing Proposition 3.5 from Proposition 4.1. Finally, Proposition 4.1 is proved in $§ 4.3$.

\subsection{Statement of Proposition 4.1.}

Proposition 4.1. Given a number field $F$, let $P(T) \in O_{F}[T]^{4}$ be a monic separable polynomial whose roots all are in $F \backslash\{0,1\}$. Then there

\footnotetext{
${ }^{4}$ As before, $O_{F}$ denotes the integral closure of $\mathbb{Z}$ in $F$.
} 
exist infinitely many positive integers $k$ such that the Galois group of $P\left(T^{k}\right)$ over $F$ has an element fixing no root of $P\left(T^{k}\right)$.

4.2. Proof of Proposition 3.5 under Proposition 4.1. Let $F$ be a number field and $P(T) \in O_{F}[T]$ a monic separable polynomial such that $P(0) \neq 0$ and $P(1) \neq 0$. Denote the roots of $P(T)$ by $t_{1}, \ldots, t_{r}$ and the splitting field of $P(T)$ over $F$ by $L$. By Proposition 4.1, there exist infinitely many positive integers $k$ such that

$$
\operatorname{Gal}\left(L_{k} / L\right) \backslash \bigcup_{j=1}^{r} \bigcup_{l=0}^{k-1} \operatorname{Gal}\left(L_{k} / L\left(\zeta_{k}^{l} \sqrt[k]{t_{j}}\right)\right)
$$

contains some $\sigma_{k}$, where $L_{k}$ is the splitting field over $L$ of $P\left(T^{k}\right), \zeta_{k}$ is a primitive $k$-th root of unity and $\sqrt[k]{t_{j}}$ is a given $k$-th root of $t_{j}$ $(j=1, \ldots, r)$. For each positive integer $k$, the splitting field of $P\left(T^{k}\right)$ over $F$ is equal to $L_{k}$. Then $\sigma_{k}$ lies in

$$
\bigcup_{j=1}^{r} \operatorname{Gal}\left(L_{k} / F\left(t_{j}\right)\right) \backslash \bigcup_{j=1}^{r} \bigcup_{l=0}^{k-1} \operatorname{Gal}\left(L_{k} / F\left(\zeta_{k}^{l} \sqrt[k]{t_{j}}\right)\right) \text {. }
$$

It then remains to use implication $(1) \Rightarrow(3)$ of Lemma 2.2 to conclude.

4.3. Proof of Proposition 4.1. We proceed by induction on the degree of the polynomial $P(T)$.

4.3.1. The case where $P(T)$ has degree 1 . Let $F$ be a number field and $t \in O_{F} \backslash\{0,1\}$. The conclusion of Proposition 4.1 for the polynomial $T^{k}-t$ easily follows from Lemmas 4.2 and 4.3 below $^{5}$.

Lemma 4.2. Assume that $t$ is a root of unity. For each number field $L$ containing $F$, there exist infinitely many integers $k \geq 1$ such that the Galois group of $T^{k}-t$ over $L$ is not trivial and each non-trivial element of the Galois group of $T^{k}-t$ over $F$ fixes no root of this polynomial.

Proof. Assume that $t$ is a primitive $N$-th root of unity. Let $L$ be a number field containing $F$ and $k$ a positive integer whose prime factors all are prime factors of $N$. As $t \neq 1$, one has $N \geq 2$ and there exist infinitely many such integers $k$. Assume that the Galois group of $T^{k}-t$ over $L$ is trivial. Then $L$ contains a primitive $k$-th root of unity, which cannot happen if $k$ is sufficiently large (depending on $L$ ). One may then assume that the Galois group of $T^{k}-t$ over $L$ is not trivial. In particular, the Galois group of $T^{k}-t$ over $F$ is not trivial either. Let

\footnotetext{
${ }^{5}$ In the case where $t$ is not a root of unity, one makes use of the following classical lemma: if a given finite group $G$ acts transitively on a given finite set $X$ with cardinality at least 2 , then there exists $g \in G$ such that $g . x=x$ for no $x \in X$.
} 
$\sigma$ be a non-trivial element of the latter Galois group. Assume that $\sigma$ fixes at least one root of $T^{k}-t$. By the definition of $k$, each root of $T^{k}-t$ is a primitive $(N k)$-th root of unity. This implies that $\sigma$ fixes each root of $T^{k}-t$, which cannot happen.

Lemma 4.3. Assume that $t$ is not a root of unity. Then $T^{k}-t$ is irreducible over $F$ for all but finitely many prime numbers $k$.

Proof. By the Capelli lemma (see e.g. [Lan02, Chapter VI, §9, Theorem $9.1]$ ), it suffices to show that, for all but finitely many prime numbers $k, t$ is not a $k$-th power in $F$. Denote the absolute logarithmic Weil height on $\overline{\mathbb{Q}}$ by $h$. Assume that there exist infinitely many integers $k \geq 1$ such that there exists $x_{k} \in F$ satisfying $t=x_{k}^{k}$. One then has

$$
h(t)=h\left(x_{k}^{k}\right)=k \cdot h\left(x_{k}\right) .
$$

As $t$ is not a root of unity and $t \neq 0$, one has $h(t) \neq 0$. Hence $F$ contains infinitely many elements each of which has height bounded by $h(t)$, which cannot happen [Nor49, Theorem 1].

4.3.2. End of the proof of Proposition 4.1. Let $r$ be a positive integer. Assume that the following condition holds:

$(\mathrm{H})$ For each number field $F$ and each monic separable degree $r$ polynomial $P(T) \in O_{F}[T]$ whose roots all are in $F \backslash\{0,1\}$, there exist infinitely many positive integers $k$ such that the Galois group of $P\left(T^{k}\right)$ over $F$ has an element fixing no root of $P\left(T^{k}\right)$.

Let $F$ be a number field and let $P(T) \in O_{F}[T]$ be a monic separable degree $r+1$ polynomial whose roots all are in $F \backslash\{0,1\}$. Denote the roots of $P(T)$ by $t_{1}, \ldots, t_{r}, t_{r+1}$. By condition $(\mathrm{H})$, there exists an integer $k_{0} \geq 1$ such that the Galois group of $\left(T^{k_{0}}-t_{1}\right) \cdots\left(T^{k_{0}}-t_{r}\right)$ over $F$ has an element $\tau$ fixing no root of this polynomial. Denote the splitting field of $\left(T^{k_{0}}-t_{1}\right) \cdots\left(T^{k_{0}}-t_{r}\right)$ over $F$ by $L$.

(a) Assume that $t_{r+1}$ is a root of unity. By Lemma 4.2, there is an integer $k_{1} \geq 1$ such that the Galois group of $T^{k_{1}}-t_{r+1}$ over $L$ is not trivial and every non-trivial element of the Galois group of $T^{k_{1}}-t_{r+1}$ over $F$ fixes no root of this polynomial. Let $\sigma$ be a non-trivial element of the former Galois group. Denote the splitting field of $T^{k_{1}}-t_{r+1}$ over $L$ by $M$ and let $\hat{\tau} \in \operatorname{Gal}(M / F)$ be a prolongation of $\tau$ to $M$.

First, assume that $\hat{\tau}$ fixes no root of $T^{k_{1}}-t_{r+1}$. Then $\hat{\tau}$ is an element of the Galois group of $\left(T^{k_{0}}-t_{1}\right) \cdots\left(T^{k_{0}}-t_{r}\right) \cdot\left(T^{k_{1}}-t_{r+1}\right)$ over $F$ fixing no root of this polynomial. Given a positive multiple $k$ of $k_{0}$ and $k_{1}$, every prolongation of $\hat{\tau}$ to the splitting field $M_{k}$ over $F$ of $P\left(T^{k}\right)$ is an element of $\operatorname{Gal}\left(M_{k} / F\right)$ fixing no root of this polynomial. Hence the desired conclusion holds. 
Now, assume that $\hat{\tau}$ fixes a root of $T^{k_{1}}-t_{r+1}$. By the definition of $k_{1}$, $\hat{\tau}$ fixes each root of $T^{k_{1}}-t_{r+1}$. Consider the element $\sigma \hat{\tau}$ of $\operatorname{Gal}(M / F)$. If $x$ denotes any $k_{0}$-th root of $t_{1}, \ldots, t_{r}$, then $\hat{\tau}(x)$ still is a $k_{0}$-th root of $t_{1}, \ldots, t_{r}$. By the definition of $\sigma$, one then has $\sigma \hat{\tau}(x)=\hat{\tau}(x)$, which is not equal to $x$ by the definition of $\hat{\tau}$. If $x$ denotes any $k_{1}$-th root of $t_{r+1}$, then, by the above, one has $\sigma \hat{\tau}(x)=\sigma(x)$, which is not equal to $x$ by the definition of $\sigma$. Hence $\sigma \hat{\tau}$ is an element of the Galois group of $\left(T^{k_{0}}-t_{1}\right) \cdots\left(T^{k_{0}}-t_{r}\right) \cdot\left(T^{k_{1}}-t_{r+1}\right)$ over $F$ fixing no root of this polynomial. As before, the desired conclusion easily follows.

(b) Assume that $t_{r+1}$ is not a root of unity. By Lemma 4.3, $T^{k_{1}}-t_{r+1}$ is irreducible over $L$ for some prime $k_{1}$. As before, denote the splitting field of $T^{k_{1}}-t_{r+1}$ over $L$ by $M$ and let $\hat{\tau} \in \operatorname{Gal}(M / F)$ be a prolongation of $\tau$ to $M$. Let $\sigma$ be an element of $\operatorname{Gal}(M / L)$ fixing no root of $T^{k_{1}}-t_{r+1}$. If $\hat{\tau}$ fixes no root of $T^{k_{1}}-t_{r+1}$, then one gets the desired conclusion as in (a) above. We may then assume that $\hat{\tau}(\alpha)=\alpha$ for some root $\alpha$ of $T^{k_{1}}-t_{r+1}$. Let $\zeta$ be a primitive $k_{1}$-th root of unity. Up to making the prime number $k_{1}$ sufficiently large (depending on $L$ ), we may assume that $L$ and $\mathbb{Q}(\zeta)$ are linearly disjoint over $\mathbb{Q}$. Then $L(\zeta) / L$ has degree $k_{1}-1$. Hence $L(\alpha)$ and $L(\zeta)$ are linearly disjoint over $L$ (as $L(\alpha) / L$ has degree $\left.k_{1}\right)$. As a consequence, the Galois group $\operatorname{Gal}(M / L(\alpha))$ is generated by some element $b$ satisfying $b(\alpha)=\alpha$ and $b(\zeta)=\zeta^{e}$ for some $e \in \mathbb{N}$. Consider the restriction $w$ of $\hat{\tau}$ to $F(\zeta)$. One has $w=c^{m}$ for some integer $m$, where $c$ is the generator of $\operatorname{Gal}(F(\zeta) / F)$ defined by $c(\zeta)=\zeta^{e}$. Then $\hat{\tau} b^{-m}$ is a prolongation of $\tau$ to $M$ which fixes each root of $T^{k_{1}}-t_{r+1}($ as $\hat{\tau}(\alpha)=\alpha)$. As in (a) above, one shows that $\sigma \hat{\tau} b^{-m}$ is an element of the Galois group over $F$ of $\left(T^{k_{0}}-t_{1}\right) \cdots\left(T^{k_{0}}-t_{r}\right) \cdot\left(T^{k_{1}}-t_{r+1}\right)$ fixing no root of this polynomial, thus ending the proof.

\section{A GeOMETRIC VARIANT}

The aim of this section is Proposition 5.1 below which makes [Leg15, Corollary 5.2] more precise (this result is recalled as Lemma 5.2 below).

\subsection{Statement of Proposition 5.1.}

Proposition 5.1. Let $G$ be a non-trivial finite group, not a cyclic $p$ group. Then there exist a number field $F_{G}$ and an $F_{G}$-regular Galois extension $E / F_{G}(T)$ with Galois group $G$ such that the following holds: (geometric non-G-parametricity) for every finite extension $F^{\prime} / F_{G}$, there exist infinitely many linearly disjoint Galois extensions of $F^{\prime}$ with Galois group $G$ each of which is not a specialization of $E F^{\prime} / F^{\prime}(T)^{6}$.

\footnotetext{
${ }^{6}$ As in the (non- $G$-parametricity) condition, the realizations of $G$ whose existence is claimed may be produced by specialization.
} 
Unlike the result in part (1) of Remark 3.2, it seems unclear whether a number field $F_{G}$ as in Proposition 5.1 may be specified for a given group $G^{7}$. See $[\operatorname{Leg} 15, \S 7]$ where this is done in some specific cases.

5.2. Proof of Proposition 5.1. Let $G$ be a non-trivial finite group.

First, recall the following result which is [Leg15, Corollary 5.2].

Lemma 5.2. There exist a number field $F_{G}$ and an $F_{G}$-regular Galois extension of $F_{G}(T)$ with Galois group $G$ which satisfies the (geometric non-G-parametricity) condition if the following group theoretic condition holds.

(H2) There exists a set $\left\{C, C_{1}, \ldots, C_{r}\right\}$ of non-trivial conjugacy classes of $G$ such that the elements of $C_{1}, \ldots, C_{r}$ generate $G$ and the remaining conjugacy class $C$ is not in the set $\left\{C_{1}^{a}, \ldots, C_{r}^{a} / a \in \mathbb{N}\right\}$.

Now, combine Lemmas 5.2 and 5.3 below to get Proposition 5.1.

Lemma 5.3. Condition (H2) fails if and only if $G$ is a cyclic p-group.

Proof of Lemma 5.3. It is not hard to see that condition (H2) fails if $G$ is a cyclic $p$-group. For the converse, we use the following argument due to Reiter. Assume that condition (H2) fails. Let $H$ be a maximal subgroup of $G$. If $H$ is not a normal subgroup of $G$, one has

$$
G=\left\langle\bigcup_{g \in G} g H g^{-1}\right\rangle \text {. }
$$

As condition (H2) fails, (5.1) provides $G=\bigcup_{g \in G} g H^{-1}$, which cannot happen. Then each maximal subgroup of $G$ is a normal one. Hence $G$ is nilpotent, i.e., $G$ is the product of its Sylow subgroups. Set

$$
G=P_{1} \times \cdots \times P_{s}
$$

with $P_{1}, \ldots, P_{s}$ the Sylow subgroups of $G$. By the Sylow theorems and as condition (H2) has been assumed to fail, (5.2) provides

$$
G=P_{1} \cup \cdots \cup P_{s} .
$$

If $s \geq 2$, then, by taking cardinalities in (5.2) and (5.3), we get

$$
\prod_{i=1}^{s}\left|P_{i}\right|<\sum_{i=1}^{s}\left|P_{i}\right|,
$$

which cannot happen. Hence $s=1$ and $G$ is a $p$-group.

Let $H_{1}$ and $H_{2}$ be two distinct maximal subgroups of $G$. Then

$$
G=\left\langle H_{1} \cup H_{2}\right\rangle \text {. }
$$

\footnotetext{
${ }^{7}$ i.e., being a regular Galois group over a given number field $F$ might not be sufficient to take $F_{G}=F$.
} 
As $H_{1}$ and $H_{2}$ are normal subgroups of $G$ and as condition (H2) fails, (5.4) provides $G=\bigcup_{g \in G} g\left(H_{1} \cup H_{2}\right) g^{-1}$. Hence $G=H_{1} \cup H_{2}$. In particular, one has $H_{1} \subseteq H_{2}$ or $H_{2} \subseteq H_{1}$, which cannot happen. Hence $G$ has only one maximal subgroup and is then cyclic, as needed.

5.3. A conjectural version of Proposition 5.1. Recall that [Leg15] also offers a conjectural version of [Leg15, Corollary 5.2]; see [Leg15, Corollary 5.3]. Below we provide a similar conjectural version of Proposition 5.1 (which then makes [Leg15, Corollary 5.3] more precise).

Namely, let $G$ be a non-trivial finite group. Assume that the following conjecture of Fried is satisfied ${ }^{8}$.

Conjecture (Fried). Each set $\left\{C_{1}, \ldots, C_{r}\right\}$ of non-trivial conjugacy classes of $G$ that is rational and such that the elements of $C_{1}, \ldots, C_{r}$ generate $G$ occurs as the inertia canonical conjugacy class set of some $\mathbb{Q}$-regular Galois extension of $\mathbb{Q}(T)$ with Galois group $G$.

Then, by combining Lemma 5.3 and [Leg15, Corollary 5.3], Proposition 5.1 holds with $F_{G}=\mathbb{Q}$, i.e., the following holds.

Proposition 5.4. Assume that $G$ is not a cyclic p-group. Then there exists a $\mathbb{Q}$-regular Galois extension of $\mathbb{Q}(T)$ with Galois group $G$ that satisfies the (geometric non-G-parametricity) condition.

5.4. Other base fields. We conclude this paper by noticing that similar statements can be given for other base fields. For example, by conjoining Lemma 5.3 and [Leg15, §5.2], we obtain the following counterpart of Proposition 5.1 for rational function fields.

Proposition 5.5. Let $G$ be a non-trivial finite group, not a cyclic $p$ group, $\kappa$ an algebraically closed field of characteristic zero and $X$ an indeterminate such that $T$ is transcendental over $\kappa(X)$. Then, for some Galois extension $E / \overline{\mathbb{Q}}(T)$ with group $G$, the extension $E \kappa(X) / \kappa(X)(T)$ satisfies the (geometric non-G-parametricity) condition.

\section{REFERENCES}

[Dèb92] Pierre Dèbes. On the irreducibility of the polynomials $P\left(t^{m}, Y\right)$. J. Number Theory, 42(2):141-157, 1992.

[Dèb01] Pierre Dèbes. Théorie de Galois et géométrie: une introduction. In Arithmétique des revêtements algébriques (Saint-Étienne, 2000), volume 5 of Sémin. Congr., pages 1-26. Soc. Math. France, Paris, 2001.

[DW08] Pierre Dèbes and Yann Walkowiak. Bounds for Hilbert's irreducibility theorem. Pure Appl. Math. Q., 4(4):1059-1083, 2008.

\footnotetext{
${ }^{8}$ See Section III.1 of http://www . math.uci.edu/ mfried/deflist-cov/RIGP. html or [Leg15, §5] for more details.
} 
[Lan02] Serge Lang. Algebra, volume 211 of Graduate Texts in Mathematics. Springer-Verlag, New York, revised third edition, 2002.

[Leg15] François Legrand. Parametric Galois extensions. Journal of Algebra, 422:187-222, 2015.

[Leg16] François Legrand. Specialization results and ramification conditions. Israel J. Math., 214(2):621-650, 2016.

[Nor49] D. G. Northcott. An inequality in the theory of arithmetic on algebraic varieties. Proc. Cambridge Phil. Soc., 45:502-509 and 510-518, 1949.

E-mail address: flegrand@post.tau.ac.il

School of Mathematical Sciences, Tel Aviv University, Ramat Aviv, Tel Aviv 6997801, IsRAel

Department of Mathematics and Computer Science, the Open UniVERSITY OF ISRAEL, RA'ANANA 4353701, ISRAEL 